\title{
Role of contrast, lettercase, and viewing conditions in a lateralized word-naming task
}

\author{
JUSTINE SERGENT \\ Montreal Neurological Institute, Montreal, Canada
}

\begin{abstract}
Because of the asymmetric functional organization of the brain, the process of naming a visually presented word necessarily engages, in its end stage, the structures of the left anterior hemisphere, forcing information received by the right hemisphere to be transferred across the corpus callosum. On the basis of this functional organization, a task requiring speeded naming of a laterally presented word was designed to examine the nature of the information transferred from the right to the left hemisphere and the type of process initially performed by the right hemisphere before transfer. Three categories of variables, each affecting particular components of the stimulus were manipulated in a within-subject design: (1) the viewing conditions, with the target word presented under flicker masking, which desensitizes the transient low-frequency channels while increasing the sensitivity of the low-frequency sustained channels, with backward pattern masking, which interferes with the integration of high spatial frequencies, or without masking; (2) the contrast of the word stimulus, which determines stimulus energy; and (3) the lettercases, which influence the relative salience of the component and configural properties of the word. Analysis of latencies showed a right-visual-field main effect and interactions between visual field and contrast and between visual field and lettercase. Right visual field superiority increased with a reduction in stimulus energy, and the right hemisphere benefited more than the left hemisphere from the additional information provided by the configural property of the lowercase word. The viewing conditions influenced the strength of these effects but did not modify their general pattern. The results are discussed with respect to interhemispheric transfer of visual information and the processing competence of the cerebral hemispheres.
\end{abstract}

The most solid evidence of functional cerebral lateralization, as well as the first to have been reported, is the almost exclusive role of the left hemisphere $(\mathrm{LH})$ in the mediation of speech production. Findings from the right-handed neurological population indicate that damage to the left frontal lobe disrupts or prevents the production of oral language while similar damage in the homotopic area of the right hemisphere (RH) has no significant effect on this process (Hécaen \& Albert, 1978). Research on commissurotomized patients has confirmed this evidence by showing that the $\mathrm{RH}$, despite language comprehension capacities, is totally mute (Sperry, 1982). More recently, inquiry into the particular processing competence of the intact hemispheres in normal subjects has corroborated these findings in experiments involving speeded verbal identification of visual stimuli such as words, pictures of objects, and colors (McKeever \& Jackson, 1979). This robust evidence of a unilateral mechanism underlying speech production suggests a convenient means of further studying the

This work was supported by a grant from the Fonds de la recherche en santé du Québec. I am grateful to Laurence Coughlin for testing the subjects and to an anonymous reviewer for helpful suggestions. Requests for reprints should be sent to Justine Sergent, Montreal Neurological Institute, 3801 University Street, Montreal, P.Q., Canada H3A 2B4. processing skills of the cerebral hemispheres, since it can be safely assumed that a task requiring speeded word-naming will engage, in its end stage, neural structures of the left frontal cortex. Thus, visual verbal information presented in the left visual field (LVF), and thus projected to the RH, must, along its elaboration and transformation in its route to the left anterior cortex, cross the midline, supposedly through the corpus callosum. The present investigation is an attempt to specify certain contributions of the cerebral hemispheres to this process and to examine some characteristics of the information that is transferred from the RH to the $\mathrm{LH}$.

Despite its apparent simplicity, producing the name of a visually presented word is a complex operation that engages perceptual, memory, and motor processes, and that can be disrupted by a lesion in various parts of the brain (Brown, 1972). Neurological evidence of a RH contribution to this operation is found only when some type of degradation, either direct or tachistoscopically induced, diminishes the perceptual quality of the input. Faglioni, Scotti, and Spinnler (1969) found that RH-damaged patients were defective relative to $\mathrm{LH}$-damaged patients in identifying fragmented letters. Rosenthal and Fedio (1975) reported that the identification of tachistoscopically presented three-letter words in the central and in the two lateral visual fields required longer 
exposure for RH-damaged patients than for LHdamaged and normal subjects. There is, however, no strong evidence of such deficits with normal viewing conditions. In fact, it seems likely that any contribution of the RH to naming visually presented words must take place at the level of visual processing, through structures that are linked to the $\mathrm{LH}$ by fibers crossing at the splenium of the corpus callosum. This is suggested by the particular deficits of patients with alexia without agraphia who typically suffer from damage to the left occipital cortex and the splenium of the corpus callosum (Geschwind, 1965). Were the RH capable of processing the incoming information beyond the visual level for mapping onto the vocalmotor system, the outcome of such processing could be transferred through the body of the corpus callosum and no alexic symptom would be apparent. This is also suggested by the performance on a wordnaming task by an epilectic patient whose corpus callosum was sectioned in two stages (Sidtis, Volpe, Holtzman, Wilson, \& Gazzaniga, 1981). After section of the posterior half of the corpus callosum, including the splenium, naming accuracy for LVF presentation dropped to chance level, although the patient could provide semantic information about the referent object when queried by the experimenter. Clearly, there must be more than the vocal-motor system that is unique to the $\mathrm{LH}$, and a purely visual code elaborated by the RH may not be sufficient as information to allow correct word production. This code must therefore be integrated by the LH-processing structures at a relatively early level if it is to contribute significantly to word-naming operations. This does not mean that the $\mathrm{RH}$ is inactive in such a process beyond the visual level, but rather that, with respect to naming per se, its contribution may no longer be critical.

In normals, the problems of interhemispheric transfer has been addressed mainly with respect to the time it takes for information to cross the corpus callosum, using the simple RT paradigm (Poffenberger, 1912). Estimates of 3 to $5 \mathrm{msec}$ have consistently been reported in simple RT tasks when a manual response was required. By contrast, contradictory results have been obtained with vocal RT estimates of transmission time, specifically when the task involved a simple vocalization to signal detection of a light flash (see Bashore, 1981, for a review). In addition, manual and vocal estimates are not equally sensitive to manipulations of the signal.

Milner and Lines (1982) recently compared manual and vocal RTs to lateralized flashes of various intensities. They observed that the time to cross the midline was invariant as a function of stimulus intensity in manual responses but increased with a decrease in stimulus intensity with vocal responses. They suggested that interhemispheric transmission in simple vocal RT tasks involved a visual code, since estimates of transfer time were inversely related to stimulus intensity. ${ }^{1}$ On the other hand, the absence of influence of signal intensity on estimates of transfer time in manual responses was considered an indication that a nonvisual code was transmitted through the corpus callosum. Moscovitch (1983) has challenged the latter interpretation and suggested that a "visual higher order" code could be transferred, since such a code would also be independent of the particular intensity of the signal. Moscovitch does not say, however, what a "visual higher order" code of a 2-msec unpatterned flash might stand for, or why such a code is not operative in the case of a verbal response.

There is, thus, a fair amount of evidence that a different code is transferred, depending on whether the response is manual or verbal. The fact that a visual code is transferred when a vocal response is required seems to follow logically from the functional organization of the cortex: If the RH has no efficient control over the motor speech system, it may not be equipped to generate the complex information necessary to activate this system, and the callosal transmission must involve a nonmotor route. While there are good reasons to believe that such a route traverses the corpus callosum at the level of visual structures, as evidenced by the influence of intensity on transfer time and by findings from brain-damaged patients, the particular nature of the information that is transmitted from the RH to the LH remains to be specified, using more complex stimuli than a simple unpatterned light: Does the RH send essentially unprocessed visual verbal information or is this information initially processed by the $\mathrm{RH}$ and then transferred? If the RH performs an initial processing of this information, is the transferred information free of intensity parameters of the signal? Does the RH outperform the LH in some aspects of this processing?

Moscovitch (1983) has recently addressed the problem of the nature of the visual code that is transferred from the $\mathrm{RH}$ to the $\mathrm{LH}$ and estimated the duration of interhemispheric transfer. He measured the interstimulus interval (ISI) necessary for a word presented in either the LVF or right visual field (RVF) to evade masking, and found that a word presented in the RVF could evade a pattern mask an average of $4 \mathrm{msec}$ sooner than a word presented in the LVF, whereas a light mask produced no visual-field difference. Moscovitch concluded from these findings that hemispheric differences emerge only at higher order central processing stages and that it takes $4 \mathrm{msec}$ for visual information to cross the corpus callosum. There are, however, two difficulties inherent in these conclusions and in the rationale underlying the experimental approach. It is doubtful that one can, at the same time, estimate interhemispheric transmission time and examine functional hemispheric asymmetry. If the two hemi- 
spheres differ in their mode of processing, such a difference must affect estimates of transmission time. More importantly, Moscovitch's (1983) experiments were not designed in a way that guaranteed transfer of information within an experimental trial. The performance criterion was identification accuracy, and naming was not under time limit. Moscovitch assumed that transfer took place immediately, before the effects of the pattern mask interfered with processing, but it is also possible that a visual representation of the stimulus was generated within the hemisphere receiving the information, and that the observed difference in critical ISI between the two visual fields reflected functional asymmetry in constructing a visual representation of the word in the respective hemispheres. Transfer may thus not have taken place immediately, and only a speeded verbal response would have imposed such a transfer within a trial. Moscovitch (1983, Experiment 1) obtained an interaction between exposure duration and visual field, indicating that interhemispheric transmission time increased with an increase in exposure duration. This result is in contrast to Milner and Lines's (1982) finding of decreasing visual-field difference with increasing stimulus intensity, but the source of this divergence remains unclear.

\section{Purpose of the Experiment}

In the present investigation, the nature of the information transferred from the RH to the LH was examined by requiring subjects to make speeded verbal identification of three-letter words presented in the LVF or RVF. Three types of variables were manipulated, and each one served the purpose of inquiring about the fate of particular characteristics of visual information in interhemispheric transfer.

Viewing conditions. The word stimuli were presented under three different viewing conditions: continuous flicker masking, backward pattern masking, and without masking. The inclusion of this variable was essentially for exploratory purposes, and no specific predictions were made with respect to the effects of each viewing condition on performance in the two visual fields.

The effect of flicker masking on the visual system is to mask selectively the transient channels without affecting the sustained channels (Breitmeyer, Levi, \& Harwerth, 1981). The transient neurons respond preferentially to low-spatial frequencies, to movement, and to the onset and of fset of stimulation. It must be noted, however, that flicker does not mask all low-frequency channels, and the sustained channels tuned to the low spatial frequencies show even greater sensitivity to these frequencies in the presence of transient masking (Legge, 1978). Thus, flicker masking allows a desensitization of the transient visual channels but does not eliminate low-frequency sensitivity of the sustained channels. In addition, transient channels are assumed to contribute little, if anything, to pattern recognition and fine discrimination (e.g., Breitmeyer \& Ganz, 1976; Lennie, 1980). Since the identification of words requires intermediateto-high spatial frequencies (Lovegrove, Heddle, \& Slaghuis, 1980), flicker masking should not significantly affect performance as compared with a viewing condition without mask. However, in lateral tachistoscopic presentations, with which the subject does not know which of two locations will be stimulated, the transient channel may play an alerting role by responding to the onset of the stimulus, which would in turn reduce uncertainty and speed the start of processing. If such is the case, one effect of flicker masking would be to lengthen $R T$ without affecting accuracy.

Backward pattern masking, on the other hand, interferes with the integration of stimulus information, particularly the higher spatial frequencies which take longer to be resolved. The effect of pattern masking should therefore be to reduce processing efficiency in terms of both speed and accuracy, because it would deprive the stimulus word of components that are critical in word identification.

Contrast. The second variable to be manipulated was stimulus energy - specifically, by using two types of display of the target words: high and low contrast. This manipulation represents another way of examining the effect of stimulus intensity on interhemispheric transmission time (cf. Milner \& Lines, 1982), and provides a comparison with Moscovitch's (1983) manipulation of stimulus energy through variation of exposure duration. If, as suggested by Milner and Lines, reduction in stimulus energy results in slower information transmission, the RT difference between visual fields should be larger with low-contrast than with high-contrast target stimuli. Such an outcome would suggest that signal intensity influences transmission time, and it would invalidate Moscovitch's assumption that interhemispheric transfer was involved in his experimental procedure. On the other hand, an absence of interaction between visual field and contrast would suggest that the information being transferred was independent of input intensity.

Type of letter. The first two variables were concerned with the intensity of incoming information and the channels that conveyed the information. The third variable to be manipulated involved structural factors, specifically, the component and configural properties of the stimulus. Garner (1978) has for a long time now argued and demonstrated that visual stimuli, such as printed words, have component (individual letters) and configural (whole-word shape) properties that coexist, the latter resulting from interrelationships among the former. Garner (1981) recently showed that there was greater configural differentiation when words were in lowercase, with ascending and descending letters (e.g., pat vs. tap) than when they were in uppercase letters of equal height (e.g., PAT vs. TAP). However, the advantage con- 
ferred by the configuration of the lowercase word over the uppercase word obtained only when the stimulus was presented under degraded conditions. Even in such conditions, Garner found that the role of individual letters as contrasted to that of configuration was predominant in a simple word-identification task, and that the configural information facilitated the identification of degraded words but did not substitute for the letter information itself. With respect to hemispheric mediation of this configural perception and processing of words, two complementary factors may be outlined. Research on commissurotomized patients (e.g., Zaidel, 1981) and on such brain-damaged subjects as deep dyslexics (e.g., Coltheart, Patterson, \& Marshall, 1980) has indicated that the RH lacks phonetic processing skills and relies on the overall visual shape of the word for identification. No study has yet directly examined this problem in word identification by normals, and, on the basis of neurological findings, one would predict that any facilitation resulting from the configural properties of lowercase words should be more pronounced in LVF than in RVF presentations, at least in the masking and low-contrast conditions. Since retinal eccentricity and brief exposure inherent in lateral tachistoscopic presentation imply some form of degradation, this prediction may extend to the nomask condition. Consistent with these predictions is the suggestion that the RH may be more adept than the LH at processing low-frequency spectral components of the incoming information (Sergent, 1982). While configural and component properties of a visual stimulus coexist, they may not be equally affected by degradation. For example, whereas a face has both component and configural properties (see Sergent, in press a), as do other multidimensional stimuli, the configural properties are more resistant to degradation than are the component properties. This is clearly illustrated by a coarse quantization of a face (such as Lincoln's, e.g., Harmon, 1973), which leaves essentially intact the configuration of the physiognomy at the same time that it totally removes the component features. Thus, a low-pass filtering of a stimulus is more detrimental to its component properties than to its configural properties, and it follows that the hemisphere that is the more sensitive to low frequencies (that is, the RH more than the LH) would be better able to use the configuration of the word in conditions that make the resolution of high frequencies difficult, such as with low contrast and a pattern mask. This is not to say that configural properties do not exist in high-quality viewing conditions, such as high contrast and without mask, but that, because they are not as informative as component letters for word identification, they are not as useful when the letters are clearly discriminable.

Thus, the experiment comprised four stimulus conditions, resulting from the orthogonal combination of two levels of contrast and two types of lettercase, with presentation in either the LVF or RVF under three different viewing conditions. Because RT was the dependent variable of interest in this experiment, the level of stimulus degradation was minimized so as to allow a high level of accuracy in order to obtain reliable latency measures. As a result, the effects of degradation were relatively weak, but they were found to be sufficiently pronounced in pilot work to produce significant variations in performance. In addition, although Garner (1981) noted that the advantage resulting from the greater configural salience of lower- as opposed to uppercase letters was stronger with words spanning less than $1 \mathrm{deg}$ of visual angle, it was necessary to use larger words because they were presented in the retinal periphery.

The use of a vocal RT word-identification task inherently implies a LH involvement, and a RVF superiority main effect was therefore expected, even though some aspects of the input were manipulated. As noted by Sergent (1983), "the characteristics of the incoming information, though still determinant, may play a secondary role (in determining hemisphere. superiority) when word identification is involved" (p. 506). Thus, relative, rather than absolute, differences between the visual fields will be of interest in examining the effects of stimulus manipulations on hemispheric processing.

\section{METHOD}

\section{Subjects}

Twelve male subjects ( 22 to 33 years of age), drawn from an unemployment agency, participated in the experiment and were paid for their time. They all had at least completed high school education. They were right-handed, as assessed with the Edinburgh Handedness Inventory (Oldfield, 1971) and a hand-tapping task, and they had no history of familial sinistrality. All had normal vision, some with correction, and all were able to name without hesitation each experimental word under the more degraded viewing condition prior to the practice trials.

\section{Stimuli}

The four words were cat, cap, tap, and pat, in upper and lower case, constructed with black Letraset LG 1313, 48-pt. letters. These letters are $12 \mathrm{~mm}$ high for upper case and lower case with descending or ascending features and $\mathbf{8 . 5} \mathrm{mm}$ for lower case, and the line thickness is $2 \mathrm{~mm}$. When projected, uppercase words were $.66 \mathrm{deg}$ high and $1.75 \mathrm{deg}$ long, with .11-deg space between adjacent letters; lowercase words were $.46 \mathrm{deg}$ high and $1.50 \mathrm{deg}$ long, with .11-deg space between adjacent letters. The inner edge of the uppercase word fell 1.5 deg from fixation. Because lowercase words were shorter by $.25 \mathrm{deg}$, and to avoid confounding effects in the comparison of visual fields and types of word, the first letter of the lowercase word was placed exactly at the same distance from fixation as the first letter of the uppercase word. This resulted in the LVF lowercase word being $.25 \mathrm{deg}$ farther from fixation than the RVF lowercase word. Pilot study had shown no significant effect of this displacement, which, in any case, would favor the RVF and would bias performance in the direction opposite to that predicted.

\section{Viewing Conditions}

The stimuli were presented through a Gerbrands tachistoscope (T-3B-1), and exposure duration was $50 \mathrm{msec}$ in all conditions. Viewing was binocular. Contrast was manipulated directly on the 
stimuli instead of superimposing a constant luminous field on the target word. The four lowercase and four uppercase words were originally printed on transparencies and then photocopied with either a white background for high-contrast words or a gray (Munsell N5) background for low-contrast words. The luminance of the background was $5 \mathrm{~mL}$ for high-contrast words and $1.12 \mathrm{~mL}$ for the low-contrast words. Defining contrast as ( $\mathrm{Lum}_{\max }-$ $\left.\operatorname{Lum}_{\min }\right) /\left(\operatorname{Lum}_{\max }+\mathrm{Lum}_{\min }\right)$, the contrast was .82 and .38 for the high- and the low-contrast words, respectively.

The flicker was a uniform field masking at a $6-\mathrm{Hz}$ temporal rate (83-msec phase), alternating between 0 and $10 \mathrm{~mL}$, and it operated continuously throughout the practice and experimental sessions of the flicker condition. The onset of the target stimulus always interrupted the on phase of the flicker, so that the word stimulus was presented during the off phase of the flicker. The pattern mask was made of random straight lines and dots that extended across the two visual fields. When superimposed on the stimulus under continuous viewing, it prevented the identification of the word. The pattern mask appeared immediately at stimulus offset and was exposed for $500 \mathrm{msec}$ at an average luminance of $5 \mathrm{~mL}$.

To ensure that only the absence or presence and type of mask differentiated the three viewing conditions, it was necessary to set the preexposure field of the no-mask and the pattern-mask conditions at the same luminance as that of the on phase of the flicker, because a preexposure field elevates the contrast threshold (Kahneman, 1965). Since a preexposure field of $10 \mathrm{~mL}$ was present in the flicker condition, this might have reduced performance in this condition for reasons other than the flicker per se. Thus, both the no-mask and the pattern-mask conditions were conducted with a preexposure field of $10 \mathrm{~mL}$ and a central dot as fixation point.

\section{Equipment}

The subject sat in front of the tachistoscope, with his head in a foreheadrest and his eyes at the level of the fixation point. A microphone was adjusted $10 \mathrm{~cm}$ in front of his mouth. The onset of the test stimulus triggered a timer (Hewlett-Packard electronic counter 5212A), which stopped at the first sound produced by the subject. The voice key was calibrated for sensitivity to each subject's voice. The RT was automatically recorded on a printer (MED Associates, DIG 900), and accuracy of response was recorded by the experimenter.

\section{Experimental Design}

Each subject was tested in three experimental sessions that corresponded to the three viewing conditions, each of 160 trials. To avoid any possible aftereffect of the flicker masking, the sessions were run on consecutive days, and the order of viewing conditions was counterbalanced across subjects. Each session was preceded by a practice session of $\mathbf{4 0}$ trials, with feedback on response accuracy. An experimental session comprised 20 trials of each of the orthogonal combination of visual field, letter type, and contrast, presented in a quasi-random order, with no more than twice the same word, four trials in the same visual field, and four words of the same letter type and of same contrast in succession. Thus, except for the viewing conditions that were blocked, all other variables were mixed within an experimental session.

\section{Procedure}

Before administration of the experiment proper, each subject answered the handedness questionnaire, performed the handtapping task, and was tested on his capacity to identify the lowcontrast words presented at $50 \mathrm{msec}$.

A trial began with the subject's being warned to fixate the central fixation dot. About $1 \mathrm{sec}$ later, a word was flashed to the right or the left of the central dot. The subject was to pronounce the name of the word loudly and distinctively, as fast and as accurately as possible. Both speed and accuracy were stressed in the instructions. A new trial occurred about every $7 \mathrm{sec}$, and an entire session lasted about $\mathbf{4 0} \mathrm{min}$, with one rest period at midpoint in the session or at the subject's request. After a rest, three practice trials were given before resumption of the experimental trials.

\section{RESULTS}

Correct latencies averaged across subjects, standard deviations, and percentages of errors are presented in Table 1, as a function of visual field, contrast, and type of letter for each viewing condition, as well as averaged across conditions.

\section{Reaction Times}

A four-way repeated-measures analysis of variance was carried out on correct latencies, the factors being viewing condition (no mask, flicker mask, and pattern mask), visual field (LVF-RVF), contrast (highlow), and type of letter (upper-lowercase). Latencies were shorter to the no-mask condition than to the

Table 1

Mean Correct Latencies (in Milliseconds), Standard Deviations (SDs), and Mean Percentage of Errors (PEs) for Each Condition of the Word-Naming Task

\begin{tabular}{|c|c|c|c|c|c|c|c|c|c|}
\hline & & \multicolumn{4}{|c|}{ Left Visual Field } & \multicolumn{4}{|c|}{ Right Visual Field } \\
\hline & & \multicolumn{2}{|c|}{ High Contrast } & \multicolumn{2}{|c|}{ Low Contrast } & \multicolumn{2}{|c|}{ High Contrast } & \multicolumn{2}{|c|}{ Low Contrast } \\
\hline & & $\begin{array}{c}\text { Upper } \\
\text { Case }\end{array}$ & $\begin{array}{l}\text { Lower } \\
\text { Case }\end{array}$ & $\begin{array}{c}\text { Upper } \\
\text { Case }\end{array}$ & $\begin{array}{l}\text { Lower } \\
\text { Case }\end{array}$ & $\begin{array}{l}\text { Upper } \\
\text { Case } \\
\end{array}$ & $\begin{array}{c}\text { Lower } \\
\text { Case }\end{array}$ & $\begin{array}{c}\text { Upper } \\
\text { Case }\end{array}$ & $\begin{array}{c}\text { Lower } \\
\text { Case }\end{array}$ \\
\hline No Mask & $\begin{array}{l}\text { Mean } \\
\text { SD } \\
\text { PE }\end{array}$ & $\begin{array}{l}651 \\
50 \\
0.10\end{array}$ & $\begin{array}{l}648 \\
54 \\
0.10\end{array}$ & $\begin{array}{l}691 \\
74 \\
1.15\end{array}$ & $\begin{array}{l}712 \\
76 \\
1.56\end{array}$ & $\begin{array}{l}646 \\
51 \\
0.00\end{array}$ & $\begin{array}{l}648 \\
46 \\
0.05\end{array}$ & $\begin{array}{c}663 \\
68 \\
0.26\end{array}$ & $\begin{array}{l}695 \\
75 \\
1.04\end{array}$ \\
\hline Pattern Mask & $\begin{array}{l}\text { Mean } \\
\text { SD } \\
\text { PE }\end{array}$ & $\begin{array}{l}664 \\
63 \\
0.05\end{array}$ & $\begin{array}{l}679 \\
72 \\
0.16\end{array}$ & $\begin{array}{l}712 \\
77 \\
1.51\end{array}$ & $\begin{array}{c}741 \\
75 \\
1.98\end{array}$ & $\begin{array}{l}651 \\
61 \\
0.00\end{array}$ & $\begin{array}{l}683 \\
57 \\
0.05\end{array}$ & $\begin{array}{l}678 \\
67 \\
0.26\end{array}$ & $\begin{array}{c}730 \\
78 \\
0.94\end{array}$ \\
\hline Flicker Mask & $\begin{array}{l}\text { Mean } \\
\text { SD } \\
\mathrm{PE}\end{array}$ & $\begin{array}{l}673 \\
62 \\
0.31\end{array}$ & $\begin{array}{l}673 \\
59 \\
0.36\end{array}$ & $\begin{array}{l}700 \\
69 \\
0.78\end{array}$ & $\begin{array}{l}714 \\
73 \\
0.78\end{array}$ & $\begin{array}{l}656 \\
53 \\
0.05\end{array}$ & $\begin{array}{l}684 \\
68 \\
0.16\end{array}$ & $\begin{array}{l}676 \\
69 \\
0.36\end{array}$ & $\begin{array}{l}711 \\
85 \\
1.25\end{array}$ \\
\hline Overall & $\begin{array}{l}\text { Mean } \\
\text { SD } \\
\text { PE }\end{array}$ & $\begin{array}{l}663 \\
58 \\
0.15\end{array}$ & $\begin{array}{l}666 \\
62 \\
0.21\end{array}$ & $\begin{array}{l}701 \\
73 \\
1.15\end{array}$ & $\begin{array}{l}722 \\
75 \\
1.44\end{array}$ & $\begin{array}{l}651 \\
55 \\
0.02\end{array}$ & $\begin{array}{l}671 \\
57 \\
0.09\end{array}$ & $\begin{array}{l}672 \\
68 \\
0.29\end{array}$ & $\begin{array}{l}712 \\
79 \\
1.08\end{array}$ \\
\hline
\end{tabular}


flicker- and pattern-mask conditions $(669,686$, and $692 \mathrm{msec}$, respectively), but this difference did not reach a reliable level of significance $[F(2,22)=2.61$, $p=.095]$. The three other main effects were significant, and, as expected, reaction times were faster to RVF than to LVF presentations [677 and $688 \mathrm{msec}$, respectively; $F(1,11)=14.64, p<.01]$. The contrast of the display had a strong influence on performance [663 and $702 \mathrm{msec}$ for high and low contrast, respectively; $F(1,11)=25.01, p<.001$ ], as had the case of the letters, since uppercase words were named faster than lowercase words [672 and $693 \mathrm{msec}$, respectively; $F(1,11)=36.27, \mathrm{p}<.001$ ].

Although the main effect of viewing condition was not significant, this factor interacted with the type of letter. As shown in Table 1, the RT difference between upper-and lowercase words was smaller in the no-mask condition (663 and $676 \mathrm{msec}$, respectively) than in the pattern- and the flicker-mask conditions [676 and $708 \mathrm{msec}$ and 676 and $695 \mathrm{msec}$, respectively; $F(2,22)=6.06, p<.01]$. In addition, there was a significant interaction of contrast $\times$ letter type $[F(1,11)$ $=26.57, \mathrm{p}<.001]$, indicating a smaller RT difference between upper- and lowercase words at high contrast ( 657 and $669 \mathrm{msec}$ ) than between upper- and lowercase words at low contrast $(687$ and $717 \mathrm{msec})$. These two interactions suggest that upper- and lowercase words were processed equally fast when the visual information was of high quality (see Table 1 , high-contrast no-mask condition), and that, due to the smaller size of lowercase letters, degradation was more detrimental to lower- than to uppercase letters. It is also noteworthy that, at high contrast, processing speed was slower in the flicker-mask than in the no-mask condition (672 and $648 \mathrm{msec}$, respectively), and, at low contrast, was slower in the pattern-mask condition than in the flicker-mask condition (700 and $715 \mathrm{msec}$, respectively). These differences approached significance in the interaction of viewing condition $x$ contrast $[\mathrm{F}(2,22)=3.35, \mathrm{p}<.053]$, and they suggest that the flicker mask was effective in reducing processing efficiency in this word-identification task.

Two interactions involving the visual fields were significant, and they are illustrated conjointly in Figure 1 . The RT difference between LVF and RVF presentations was smaller at high contrast [665 and $661 \mathrm{msec}$, respectively] than at low contrast [712 and $692 \mathrm{msec}$, respectively; $\mathrm{F}(1,11)=6.53, \mathrm{p}<.05$ ]. This suggests that the speed with which information was transferred from the RH to the LH was a function of the sensory quality of the input, which concurs with a similar finding by Milner and Lines (1982). The other significant interaction was between visual field and type of letter $[F(1,11)=18.88, p<.002]$, and it showed that the increase in RT between upper- and lowercase words was larger for RVF presentations (662 and $692 \mathrm{msec}$, respectively) than for LVF presentations (682 and $694 \mathrm{msec}$, respectively). Thus, compared with uppercase words, the RH was processing lowercase

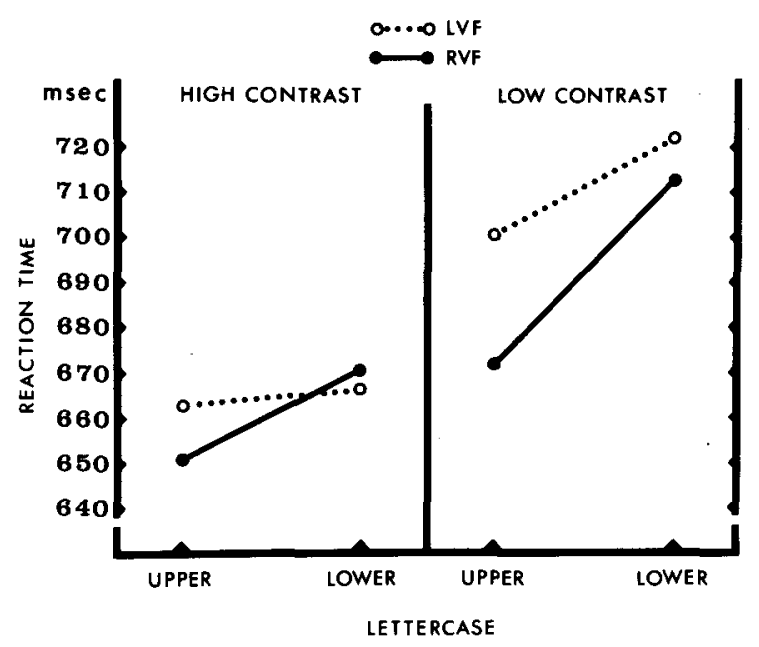

1. Mean latencies (in milliseconds), averaged across subjects, to name a word presented in the left visual field (LVF) or right visual field (RVF) as a function of contrast and lettercase.

words faster than was the LH. This finding is clearly illustrated with high-contrast words (see Figure 1) that were named more quickly after LVF than after RVF presentations despite the fact that information had to be transferred through the corpus callosum. The same trend is apparent with low-contrast words, but, because of reduced intensity of the incoming information, transfer time was longer, which contributed to the faster naming of words presented in the RVF. Since the effect was essentially similar at both contrast levels, the interaction between visual field, type of letter, and contrast was not significant $(F<1)$.

This relative advantage of LVF over RVF presentations with lowercase words may be attributed to the configural properties of the word which are more salient with lower- than with uppercase letters (Garner, 1981), and which the RH may use more efficiently than the LH. This interaction may then indicate that some "cognitive" processing of the visual word was performed within the RH before information was transferred to the $\mathrm{LH}$, since using the configuration of the word as an aid for identification must call for some knowledge about the visual structure of the word, beyond the sensory level. The two interactions involving the visual fields and suggesting that signal intensity affects transmission time for one and that configural information is transferred for the other suggest that the distinction between these two levels of information processing may not be altogether relevant. This will be discussed later.

No other interaction was significant, and additional analyses were performed on each viewing condition separately to examine in greater detail the contribution of the absence or presence and type of mask to the obtained effects. The main effects of letter type and contrast were significant in each viewing condition at the .01 level. The main effect of visual field was reliable in the no-mask and pattern-mask 
conditions $(p<.05)$, but not in the flicker-mask condition [LVF, $690 \mathrm{msec}$; RVF, $682 \mathrm{msec} ; \mathrm{F}(1,11)=$ $1.68, \mathrm{p}>.20]$.

The two interactions that involved the visual fields and were significant in the main analysis were also significant in the pattern-mask condition, with the interaction between visual field and type of letter being highly reliable $[F(1,11)=52.46]$. By contrast, only the interaction between visual field and type of letter reached significance in the flicker condition $[F(1,11)=6.44, \mathrm{p}<.05]$, while only that between visual field and contrast was significant in the nomask condition $[F(1,11)=4.95, p<.05]$. The absence of a significant interaction of visual field $x$ letter type in the no-mask condition $[F(1,11)=2.99]$ suggests that the relative advantage to the $R H$ resulting from the configural shape of lowercase words requires sufficient degradation to be effective.

\section{Errors}

The number of errors was small, a total of 255 out of 5,760 trials $(4.43 \%)$; they were analyzed with the same four-way analysis of variance as were the latencies. The results of this analysis paralleled those obtained in the analysis of RTs, with two exceptions. Only one effect that was significant with latencies was not reliable with errors, namely the interaction between visual field and letter type $[F(1,11)=1.80]$, although the trend was similar, showing a greater difference in accuracy between upper- and lowercase words in the RVF $(.31 \%$ and $1.16 \%$, respectively) than in the LVF $(1.30 \%$ and $1.65 \%$, respectively).

The other difference between latencies and errors involved the flicker condition. Two interactions involving the viewing conditions and the visual fields, which were not significant with latencies, proved reliable with errors. One was the two-way interaction between these two factors $[F(2,22)=3.89, p<.05]$, which was explained by the three-way interaction between these two factors and contrast $[F(2,22)=14.79$, $\mathrm{p}<.001]$, and which showed that the flicker mask produced fewer errors than did the other two conditions in LVF presentations at low contrast but more errors than the other conditions in RVF presentations at high contrast (see Table 1). Thus, except for a different pattern of results for latencies and errors in the flicker condition, the analysis of errors confirmed that of latencies, and there was no speedaccuracy tradeoff.

\section{DISCUSSION}

Because of the asymmetric functional organization of the brain, a word-identification task engages, in its end stage, the neural structures of the LH, forcing information received by the $\mathrm{RH}$ to cross the corpus callosum at some point between the reception of vi- sual input and the production of the motor vocal command. The results of this experiment provide some indications concerning the nature of the transferred information and of the processing taking place in the $\mathrm{RH}$ before the transfer. The results will first be discussed with respect to the two main variables of interest, contrast and letter type. The particular influence of the viewing conditions will be considered thereafter.

\section{Interhemispheric Transfer}

The transfer of information across the corpus callosum takes time and requires at least one more synapse than does a direct route within the same hemisphere, and this was reflected in the main effect of the visual field's showing faster naming when processing could be performed by the LH from input reception to response production. The difference in RT between the two visual fields was not invariant as a function of manipulations of the input, however, and it increased with a reduction in stimulus energy. Thus, reduced stimulus contrast slowed down not only the transmission of neural information in the visual structures of the two hemispheres but also that across the hemispheres, as suggested by the main effect of contrast and the interaction of contrast and visual field. These findings are identical to those reported in a previous study when reduction in stimulus intensity was produced directly (Milner \& Lines, 1982). On the other hand, they depart from Moscovitch's (1983) finding that visual field differences increase with an increase in stimulus energy (exposure duration). As noted in the introduction, Moscovitch's procedure does not guarantee that interhemispheric transfer was taking place within an experimental trial, and his finding may in fact be more consistent with the suggestion of differential hemispheric sensitivity to variations in exposure duration (see Sergent, 1982).

Milner and Lines (1982) interpreted the interaction between visual field and intensity as evidence that the information transferred across the corpus callosum was intensity-dependent, since otherwise transfer time would have been invariant as a function of stimulus manipulation. While such an interpretation may follow directly from Milner and Lines's use of a flash of light as stimulus, it would be too simple as an account of the present finding because it would imply that all the information received by the RH would be immediately transferred to the LH. Indeed, the significant interaction between visual field and lettercase indicates that some processing of the information received by the RH was carried out within this hemisphere before the information was transferred to the LH. The ascending and descending features of letters composing lowercase words provided greater configural differentiation among the words than did 
uppercase letters, and the RH could benefit more than the LH from this additional information for word identification. One effect of this greater benefit for the RH was that the naming of lowercase words presented at high contrast was faster in the LVF than in the RVF (see Figure 1), that is, when stimulus energy was high and allowed fast neural transmission across the hemispheres. The same relative benefit for the RH was found at low contrast with lowercase compared with uppercase words, but it manifested itself differently due to low stimulus intensity which slowed down transmission from the RH to the LH. These patterns of results confirm the important demonstration by Hellige (1980) that the overall speed of processing in each hemisphere can be decomposed into several subprocesses, the efficiency of which varies differentially between the hemispheres. They also show that equal overall performance in the two visual fields is no guarantee of similar underlying processes in the two hemispheres, contrary to frequent conclusions in research on cerebral functional lateralization.

These findings indicate that some configural processing of the word was performed in the RH before transfer. Yet, the duration of transfer was influenced by stimulus intensity, which suggests that, even though information is processed in the RH beyond the purely sensory level, the code being transferred is not free from the influence of the intensity levels of the input. This is not consistent with the usual distinction between sensory (or lower order) and cognitive (or higher order) levels of visual information (e.g., Moscovitch, 1983). As noted by Sergent (1983), such a distinction may be only artifactual, and recent anatomical and physiological evidence with respect to the organization of primates' visual cortical areas suggests that considerably more space than previously thought is devoted to "lower order" processing in the brain (see Sergent, in press c). Cortical areas outside the so-called "primary visual sensory area" are involved in the processing of specific components of the visual input, and the output of their operations contains information about these characteristics (e.g., Merzenich, 1982). Thus, variations in input intensity may still affect the processing of information beyond the striate cortex, and this is confirmed by electrophysiological recordings in monkeys. For example, Rolls, Perrett, Caan, and Wilson (1982) studied the activity of specific neural cells in the medial temporal cortex and the anterior thalamus in response to presentation of novel or familiar stimuli. They found that cells were activated differently, depending on whether or not the stimulus had been seen earlier, and that their particular firing was correlated with behavioral responses to novelty or familiarity. They further manipulated stimulus intensity by reducing the duration of stimulus exposure from 1,000 to $100 \mathrm{msec}$, and this manipulation modi- fied the amplitude of cell discharge, suggesting that, even in areas underlying pattern recognition, sensory parameters such as stimulus intensity, though not critical for recognition, contribute to the particular pattern of cell activity. This may indicate that the distinction between lower and higher order information may not be altogether relevant in specifying the nature of the visual code that is transferred, at least with respect to complex information such as the printed words used in this experiment.

It would therefore seem that the code being transferred from the RH to the LH contains visual information which has already been subjected to some configural processing but which is nonetheless still dependent on particular intensity levels of the input.

\section{Influence of Viewing Conditions}

Compared with the absence of mask, the two mask conditions yielded longer latencies, but this increase in RTs was only marginally significant in the main analysis. As noted in the introduction, the detrimental effect of masking was intentionally kept at a low level to ensure reliable latency measures, and the trends found in the present results suggest that more severe masking might produce significant differences that could be attributed to selective desensitization of particular channels of the visual system. While backward pattern masking is a common procedure in visual perceptual research (e.g., Eriksen \& Schultz, 1978), flicker masking has seldom been used in research on pattern recognition and word identification. The present results, although not entirely conclusive, point to a role for the transient channels (that are selectively made less responsive by flickering light, e.g., Breitmeyer et al., 1981) in lateral tachistoscopic presentations. As proposed by Breitmeyer and Ganz (1976), the transient channels may play a role in initiating selective attention to locations in visual space that contain significant figural information, and such a function may be of importance when stimuli are randomly presented in either visual field. The longer latencies obtained in the flicker condition than in the no-mask condition could then reflect some delay in orienting attention to the side of stimulation.

The separate analysis of RTs under flicker masking revealed an absence of visual-field main effect, in contrast to the other two conditions, showing that words presented in the RVF were not named significantly faster than words presented in the LVF. Given the necessity for information received by the RH to be transferred to the $\mathrm{LH}$, this finding indicates that the flicker mask was relatively less detrimental to $\mathbf{R H}$ than to $\mathbf{L H}$ processing. One account of this finding would suggest that the $\mathrm{LH}$ is normally more sensitive than the RH to the information conveyed by the transient channels, and that making these channels less responsive slows down processing more in the $\mathrm{LH}$ than in the RH. Such an interpretation would call into 
question the suggestion that the RH is more sensitive than the LH to low spatial frequencies (Sergent, 1982). There is, however, reason to believe that the present results do not contradict Sergent's suggestion. Low spatial frequencies are processed by both transient and sustained channels and, as demonstrated by Legge (1978), while transient channels are affected by flicker masking, sustained channels are not, and they even show greater sensitivity to low frequencies in such conditions. In the processing of patterned information, such as visually presented words, the relevant figural informaton is carried by the sustained channels, which therefore play a critical role in the mediation of input processing. Thus, the hemisphere that is the more sensitive to the low frequencies conveyed by the sustained channels should be the more efficient at processing the configural properties of the word that are more prevalent in the low spatial frequencies. As shown in Table 1, it was under the flickermasking condition that the advantage conferred by the configuration of the lowercase words was stronger for LVF than for RVF presentations, whereas no such relative facilitation was observed with uppercase words. This suggests that the absence of RVF superiority in the flicker condition cannot be attributed to a slowing down of $\mathrm{LH}$ processing due to desensitization of the transient channels, which should have affected both upper- and lowercase words, but can be attributed to a relative benefit of $\mathrm{RH}$ processing resulting from greater sensitivity of the low-frequency sustained channels. This interpretation is supported by the absence of interaction between visual field and contrast in the flicker condition, showing that the RT advantage of RVF over LVF presentations did not increase as much with reduction in stimulus energy as in the other conditions. It must be noted, however, that these effects had not been specifically predicted, and further investigation is necessary to ascertain the validity of their interpretation.

The pattern mask produced a very strong interaction between visual field and lettercase, but this interaction was not significant in the no-mask condition. One of the effects of the backward pattern mask is interference with the integration of the highfrequency components of the stimulus which take longer to be resolved; this effect is more pronounced when contrast is reduced (e.g., Breitmeyer \& Ganz, 1976; Kitterle \& Rysberg, 1976). Thus, with a pattern mask, the configuration more available in the lowercase words than in the uppercase ones may have become more prominent since the individual letters were less discernible as a result of reduction in highfrequency components and the RH could use this configuration more efficiently than could the $\mathrm{LH}$. This does not mean that the LH cannot process the configural properties of a visual stimulus; it is simply less efficient than the RH in the presence of degraded information, specifically when this informa- tion is deprived of its high-frequency components. In fact, there is evidence that the $\mathrm{LH}$ is equipped to process visual stimuli such as faces in terms of their configural properties, and that it may even prove better than the RH when highly contrasted information is presented (Sergent, in press b). In this respect, it is noteworthy that the advantage conferred to the $\mathrm{RH}$ by the configural properties of lowercase words was not significant in the no-mask condition, confirming Garner's (1981) suggestion that it requires sufficient degradation for these properties to be of benefit in a word-identification task for which individual letters are more informative and constitute the primary basis.

\section{CONCLUSION}

The results of this experiment suggest that information received by the $\mathrm{RH}$ is subjected to processing at several levels before being transferred to the $\mathbf{L H}$ for further transformation into codes that will activate the vocal-motor system. The finding that $R T$ differences between visual fields vary with stimulus contrast and with the particular processing implemented depending on stimulus properties indicates that estimates of transmission time using words as stimuli are likely to be unreliable and to vary as a function of experimental variables. Since some processing is carried out in the RH before transfer, at a speed different from that in the LH, RT differences between the visual fields cannot simply reflect the duration of interhemispheric transfer and are the product of more than one factor.

While the results confirmed a relative superiority of the RH in visual processing of degraded information, they also suggested that the nature of the degradation was an important factor in determining the processing efficiency of the two hemispheres. Although the different effects associated with the two types of mask were weak, they nonetheless point to a differential sensitivity of the hemispheres to the information conveyed by the various channels of the visual system. Further inquiry into the asymmetric effects of different types of masking on hemispheric processing may then be useful to examine the particularities of the neural representations elaborated in each hemisphere and the constraints these representations impose on the nature of the processing that each hemisphere can implement.

\section{REFERENCES}

BAshore, T. R. (1981). Vocal and manual reaction time estimates of interhemispheric transmission time. Psychological Bulletin, 89, 352-368.

Breitmeyer, B., \& GANz, L. (1976). Implications of sustained and transient channels for theories of visual masking, saccadic suppression, and information processing. Psychological $R e$ view, 83, 1-36. 
Breitmeyer, B., Levi, D., \& Harwerth, R. (1981). Flicker masking in spatial vision. Vision Research, 21, 1377-1385.

Brown, J. W. (1972). Aphasia, apraxia, and agnosia. Springfield, IL: Thomas.

Coltheart, M., Patterson, K., \& Marghall, J. C.: (Eds.) (1980). Deep dyslexia. London: Routledge and Kegan Paul.

Eriksen, C. W., \& Schultz, D. W. (1978). Temporal factors in visual information processing. In J. Requin (Ed.), Attention and performance VII. New York: Academic Press.

Faglioni, P., Scotti, G., \& Spinnler, H. (1969). Impaired recognition of written letters following unilateral damage. Cortex, $5,120-133$.

Garne R, W. R. (1978). Aspects of a stimulus: Features, dimensions, and configurations. In E. Rosch \& B. B. Lloyd (Eds.), Cognition and categorization. Hillsdale, $\mathrm{NJ}$ : Erlbaum.

Garne R, W. R. (1981). The role of configuration in the identification of visually degraded words. Memory \& Cognition, 9, 445-452.

Geschwind, N. (1965). Disconnection syndromes in animals and man. Brain, 88, 585-644.

HARMON, L. (1973). The recognition of faces. Scientific American, 227, 71-82.

Hécaen, H., \& Albert, M. L. (1978). Human neuropsychology. New York: Wiley.

Heltioe, J. B. (1980). Effects of perceptual quality and visual fields of probe stimulus presentation on memory search for letters. Journal of Experimental Psychology: Human Perception and Performance, 6, 639-651.

Kahneman, D. (1965). Exposure duration and effective ground contrast. Quarterly Journal of Experimental Psychology, 17, 308-314.

Kitterle, F. L., \& Rysberg, J. A. (1976). The effect of exposure duration on the apparent contrast of sinusoidal gratings. Perception \& Psychophysics, 19, 33-39.

LEGGE, G. E. (1978). Sustained and transient mechanisms in human vision: Temporal and spatial properties. Vision Research, 18, 69-81.

Lennie, P. (1980). Parallel visual pathways: A review. Vision Research, 20, 561-594.

Lovegrove, W. J., Heddle, M., \& Slaghuis, W. (1980), Reading disability: Spatial frequency specific deficits in visual information store. Neuropsychologia, 18, 111-115.

McKeever, W. F., \& JAckson, T. L. (1979). Cerebral dominance assessed by object- and color-naming latencies: Sex and familial sinistrality effects. Brain and Language, 7, 175-190.

Merzenich, M. M. (1982). Organization of primate sensory forebrain structures: A new perspective. In $\mathbf{R}$. A. Thompson \& J. R. Green (Eds.), New perspectives in cerebral localization. New York: Raven Press.

Milner, A. D., \& Lines, C. (1982). Interhemispheric pathways in simple reaction time to lateralized light flash. Neuropsychologia, 20, 171-179.

Moscoviтch, M. (1983). Laterality and visual masking: Interhemispheric communication and the locus of perceptual asymmetries for words. Canadian Journal of Psychology, 37, 85-106.
OldFiELD, R. C. (1971). The assessment and analysis of handedness: The Edinburgh inventory. Neuropsychologia, 9, 97-113.

Poffenberger, A. T. (1912). Reaction time to retinal stimulation with special reference to the time lost in conduction through nerve centers. Archives of Psychology, 23, 1-73.

Rolls, E. T., Perrett, D. I., CaAn, A. W., \& Wilson, F. A. W. (1982). Neuronal response related to visual recognition. Brain, 105, 613-646.

Rosenthal, L. S., \& Fedio, P. (1975). Recognition thresholds in the central and lateral visual fields following temporal lobectomy. Cortex, 11, 217-229.

SERgENT, J. (1982). Theoretical and methodological consequences of variations in exposure duration in visual laterality studies. Perception \& Psychophysics, 31, 451-461.

Sergent J. (1983). The role of the input in visual hemispheric asymmetries. Psychological Bulletin, 93, 481-512.

Sergent, J. (in press a). An investigation into component and configural processes underlying face perception. British Journal of Psychology.

Sergent, J. (in press b). Configural processing of faces in the left and the right cerebral hemispheres. Journal of Experimental Psychology: Human Perception and Performance, 10.

Sergent, J. (in press c). Inferences from unilateral brain damage about normal hemispheric functions in visual pattern recognition. Psychological Bulletin.

Sidtis, J. J., Volpe, B. T., Holtzman, J. D., Wilson, D. H, \& Gazzaniga, M. S. (1981). Cognitive interaction after staged callosal section: Evidence for transfer of semantic activation. Science, 212, 344-346.

SPERRY, R. W. (1982). Some effects of disconnecting the cerebral hemispheres. Science, 217, 1223-1226.

ZAIDEL, E. (1981). Reading by the disconnected hemisphere: An aphasiological perspective. In Y. Zotterman (Ed.), Wenner Gren Symposium on Dyslexia. London: Plenum.

\section{NOTE}

1. It must be noted, however, that Milner and Lines (1982) discarded half the subjects tested in the first part of the vocal task because these subjects failed to show a right-visual-field advantage. This raises questions about the validity of the interpretation proposed by Milner and Lines, since it is unlikely that the 12 righthanded subjects who showed LVF superiority in vocal RT had speech-production mechanisms represented in the RH. It is therefore possible that the simple RT task with a lateralized flash does not involve transfer across the corpus callosum, especially since in such a task, in which no figural information has to be processed, subcortical structures may play an important role. There is as yet no empirical evidence that would allow clarification of this issue, and Milner and Lines's suggestion should therefore be accepted until disproved.

(Manuscript received December 5, 1983; revision accepted for publication February 20, 1984.) 